\title{
The Dental Role in Forensic Medicine.
}

\author{
${ }^{1}$ Dr.Aruna Kanaparthy, ${ }^{2}$ Dr.Rosaiah Kanaparthy, \\ ${ }^{1}$ Reader, Conservative Dentistry \&Endodontics, Peoples Dental Academy,Bhopal,Madhya Pradesh. HIG- \\ ${ }^{3}$ PDA Staff Quarters, Peoples Campus, Bhanpur,Bhopal \\ ${ }^{2}$ Professor, \& HOD.Dept Of Periodontics, Peoples Dentalacademy,Bhopal,Madhya Pradesh HIG-3,PDA Staff \\ Quarters, Peoples Campus,Bhanpur,Bhopal.
}

\section{INTRODUCTION}

Identification of individuals is not always straightforward and easy especially in medicolegal cases where the individuals are either unable to give accurate answers or are purposefully misleading.Living individuals for whom identification is required are criminals attempting to elude custody,amnesia victims, comatose victims, victims of disfiguring trauma or persons requiring identity confirmation following identity theft.Deceased individuals requiring identity confirmation maybe the homeless,illegal/undocumented immigrants,burnt bodies or decomposed,disfigured,skeletal remains of individuals ${ }^{1}$. The most common methods for establishing a positive identification are visual,fingerprint,DNA and dental comparison ${ }^{2}$. Forensic odontology is the combination of the art and science of dentistry and the legal system,a crossroads of dental science and law.In a dental identification,the initial goal of the forensic dentist is to obtain a set of postmortem photographs,radiographs and accurate dental charting After the postmortem charting photography and radiography,the antemortem records are procured.Identification of the deceased person is carried out by comparing postmortem and antemortem records once a tentative identity of the person is known.Dental records are sought from dental practitioners or other dental facilities such as dental teaching facility, trauma centres or hospital-based dental programs. This article is an overview of the various methods of establishing the identity of an individual from the dental perspective.

The need to identify human remains ${ }^{3}$ :Medicolegal cases/crime scenes need to be resolved. Monetary matters such as life insurance and pension can be cleared only after the identification of the deceased. Religious beliefs make it mandatory to identify individuals before burial. Legal settlement of estates requires a death certificate. Positive identification of the dead individuals is necessary to reduce the agony of uncertainty of family members. Staging of death for fraudulent purposes requires forensic investigation. Society's duty to preserve human rights and dignity beyond life begins with the basic premise of an identity.

\section{METHODS TO IDENTIFY THE REMAINS OF A PERSON}

Confirm that the remains are that of a human by a forensic pathologist and anthropologist failing which a DNA analysis can be carried out by isolating the human telomerase gene on chromosome 5 which is specific for humans. Establishing identity by visual,fingerprint,DNA and dental comparison. Circumstantial evidence is that evidence found at the site of remains such as the location,personal items clothing,jewellery,cell phone etc.External characteristics such as sex weight height,skin color,hair,tattoos,scars,birthmarks,etc. Internal characteristics revealed by autopsy such as cancer,heart disease,lung disease,gall stones,kidney stones,implants,suture material,etc. Radiographs to identify bony characters such as fractures, deformities presence of foreign bodies such as bullets, shrapnel,surgical hardware, evaluation of age and growth by presence or absence of growth plates and the extent of osteophyte formation etc. Forensic anthropology is useful in cases of decomposed or skeletal remains and can be used to identify the sex,race,ancestry and stature of individuals. Cranio-facial reconstruction (CFR) ${ }^{4}$ When there is a body that is unrecognisable due to its state of decomposition, skeletisation, mutilation or calcination, a cranio-facial reconstruction (CFR) can be considered to recreate the face of the individuals immediately prior to their death also computer software and several 3D manual methods are available for this purpose.

\section{CHEILOSCOPY}

Lip prints are similar to fingerprints,palm prints and footprints in that individual characteristics are used for identification. The creases on the vermilion border of the lips, which appear as white areas in lip prints, and the raised reddish areas outlined by these creases, which appear as dark areas, are analogous to the furrows and ridges of friction ridge skin. The creases on the vermilion border are also referred to as grooves, furrows, wrinkles and valleys.Lip prints are unique and do not change during the life of a person. It has been verified that 
they recover after undergoing alterations like trauma, inflammation and diseases like herpes and that the disposition and form of the furrows does not vary with environmental factors. The lip prints of parents and children and those of siblings have shown some similarities. It has also been suggested that variations in patterns among males and females could help in sex determination.

\section{FORENSIC ODONTOLOGY:}

The durability of the human dentition and the ability of teeth to survive decomposition makes dental evidence one of the most reliable,efficient and accurate methods of identification.that it is also cost-effective and less time consuming are added advantages of forensic dentistry. Things about the individuals lifestyle can be determined by the teeth; a constant pipe smoker or a bagpiper player has a distinctive wear pattern. In addition to the dental records, forensic investigators can retrieve DNA samples not only by extracting the pulp from the center of the tooth but also from root canal treated teeth,that is from the calcified tissues ${ }^{6}$.

\subsection{Bite marks ${ }^{7}$}

Bite marks are an important aspect of forensic odontology. Bite injuries are generally seen in crimes such as fights between young children,hand-to-hand mortal combat between adults and in sex crimes. In human bites, a bite mark must be recognized and registered early, as the clarity and shape of the mark may change in a relatively short time in both living and dead victims. Bite marks appear most often as elliptical or round areas of contusion or abrasion, occasionally with associated indentations. There may be avulsion of tissue, or even pieces of tissue bitten off. There may be considerable bruising and wounds that have penetrated the skin. According to "Frye test." the requirement for

admissibility in the court of law has three components:

[1] the principle must be demonstrable;

[2] it must have been sufficiently established;

[3] it must have gained the general acceptance of experts working in the particular scientific field(s) to which the evidence belongs.

Once the mark is initially evaluated, it should be examined by a forensic odontologist to determine if the dimensions and configuration are within human ranges. Since most individuals secrete the ABO blood groups in their saliva,swabbing the area and a control area elsewhere on the body should be carried out before the body is washed. The swabs should be allowed to air dry and then submitted to a serological laboratory.Bite mark dusting and photography are the primary means of recording and preserving the bite mark and the accuracy of recording is critically important in documenting the bite mark as bite mark analysis can become controversial in nailing the perpetrator. Generally bite mark photographs are taken with reference scale developed by members of the American Board of Forensic Odontology.The significance of the nature of light and camera chosen for the purpose cannot be overemphasized as they must satisfy the rigorous scrutiny and analysis of experts. When comparing the "fingerprint powder lift" technique against the photographs, the use of photographs resulted in higher scores determined by a modified version of the ABFO scoring guidelines. The use of transparent overlays is considered subjective and irreproducible because the tracing can be easily manipulated. On the other hand, photocopier-generated overlays where no tracing is used is considered to be the best method in matching the correct bite mark to the correct set of models without the use of computer imaging.

While the photocopier-generated technique is sensitive, reliable, and inexpensive, new methods involving digital overlays have proven to be more accurate. Two recent technological developments include the 2D polyline method and the painting method. Both methods use Adobe Photoshop. Use of the 2D polyline method entails drawing straight lines between two fixed points in the arch and between incisal edges to indicate the tooth width. Use of the painting method entails coating the incisal edges of a dental model with red glossy paint and then photographing the model. Adobe Photoshop is then used to make measurements on the image. A total of 13 variables were used in analysis. Identification for both methods were based on canine-to-canine distance ( 1 variable), incisor width (4 variables), and rotational angles of the incisors ( 8 variables). The 2D polyline method relies heavily on accurate measurements, while the painting method depends on precise overlaying of the images. Although both methods were reliable, the 2D polyline method gave efficient and more objective results. To preserve the three-dimensional nature of the bitten area, impressions can be taken and stone models fabricated. In addition to these, intraoral photographs and bite impressions can be secured from the victims and potential suspects. In addition to these dental radiographs and microbiologic cultures may prove to be useful. Role of nanotechnology in forensic analysis ${ }^{8}$ In many cases, forensic evidence is key to obtain a conviction and often only very small quantities of forensic material are found on a suspect.Nanotechnology plays an important role in addressing current concerns. It can be applied to forensic trace evidence analysis as follows. 
- Post blast explosive residues analysis

- Gun-shot residue analysis

- $\quad$ DNA Analysis- In crime cases,DNA analysis of blood stains, hairs, fibres ,semen,tooth structures,saliva samples etc can be carried out.

- $\quad$ Physical clue materials- Paint and other protective coatings such as lacquer, enamel varnish frequently recovered in hit and run, burglary and forced entry cases,label comparison can be analyzed by applying nano-techniques.

- $\quad$ Fire and arson cases where hydrocarbon residues are needed to be analyzed.

- Cyber Forensics, Tape Authentication and speaker identification(by identifying

the nano coating material,the authentication of tapes can be determined). The ion beam analysis is a group of techniques which can be used to study forensic materials.

\subsection{Steps in dental identification ${ }^{1,2,3}$}

The philosophy of dental identification is that postmortem dental records can be compared with antemortem dental records, written notes,study casts,radiographs,photographs and dental charts to confirm identity. Obviously, individuals with extensive dental treatments are easier to identify than those individuals with little or no dental treatment. Once an unidentified body is located, tentative identification is carried out and the individuals antemortem records are procured from available dental facilities. Meanwhile the postmortem dental records are prepared by locating and documenting anatomical structures,restorations,appliances etc and complete dental charting is done.Close-up photographs can provide the ability to view specific and relevant features without the need to revisit the morgue.A full series of radiographs are to be taken with attention to clarity and details so that complete radiographic information is available for comparison to antemortem data. The postmortem records whether digital or paper,should be recorded in a form that will assist in the comparison process.It should be a format that records and displays the relevant features of the dentition to demonstrate missing teeth and the restored surfaces.

Securing antemortem records can be the biggest challenge for the investigating agencies and the quality of these records is totally dependent on practicing dentists.Digital duplication of these records can be carried out and the original records returned to the dentists.It is important to review all the written records, the patient ledgers , any procedural notes and radiographs till the latest known dental status of the individual. After the postmortem records and antemortem records are completed,the comparison process begins. This can be carried out manually or assisted by a computer program such as WinID3.The search results are displayed in five separate tables as most dental hits,least dental mismatches,most restoration hits, most identifier matches,and fuzzy dental logic.

According to the American Board of Forensic Odontology the following conclusions can be drawn from the identification $^{1,2}$

- $\quad$ Positive identification: The antemortem and postmortem data match in sufficient

detail,with no unexplainable discrepancies, to establish that they are from the same individual.

- Possible identification: the antemortem postmortem data have consistent features but, because of the quality of either the postmortem remains or the antemortem evidence, it is not possible to establish identity positively.

- Insufficient evidence: The available information is insufficient to form the basis for a conclusion.

- Exclusion: the antemortem and postmortem data are clearly inconsistent.

\subsection{Age estimation using dental data ${ }^{9,10}$}

Dental age estimation is important not only for identification of the deceased victims but also in connection with crimes,accidents,legal matters,school attendance,social benefits, records,employment and marriage. Dental age estimation in children combines morphological and radiological evaluation in the atlas system as advocated by Schour and Massler,Moorrees et al and Anderson et al.Scoring system by Demirjian et al attempts to simplify age estimation by restricting the analysis to 7 teeth of the lower left quadrant giving a graphical presentation of 8 stages and giving a score of A through $\mathrm{H}$. Dental age estimation in adults uses the morphological analysis of age changes as suggested by Gustafson and others which evaluate occlusal attrition,periodontosis, secondary dentin formation,cemental apposition,root resorption,root transparency and cemental annulations. The methods used for age determination depend on age related morphological changes of the tooth which can be quantified by radiology or more accurately by $3 \mathrm{D}$ digital tooth imaging. Cone-beam CT scanning provides us a new method to acquire the $3 \mathrm{D}$ images of teeth in living individuals. Using their 3D images the ratio of pulp/tooth volume can be calculated and the age determined. Cemental incremental lines 11,12 
Age estimation by counting incremental lines in cementum is considered an acceptable method by some authors. Countable cemental annulations are present in human teeth. Cross-sections through undecalcified tooth roots can be properly stained and mounted so that cemental annulations can be photographed through a light microscope.Annulations counted from a photograph provide a close estimate of the actual age of the individual from which the tooth was extracted. This technique may be extremely valuable in forensic medicine, forensic dentistry, and anthropology.However if there is periodontal disease or there are areas of root resorption this method is not valid for age estimation. Height Estimation by tooth dimensions ${ }^{13}$ Height is a useful element in human identification and its estimation is not an easy task. The stature of the subject can still be estimated based on the proportionality with tooth dimensions. The Carrea's index for stature estimation is a convenient, simple and inexpensive method, and can provide valuable information to the forensic investigation when dental remains are present.

\section{CONCLUSION}

In forensic matters, the significance of antemortem records cannot be overemphasized as it has a great impact on the lives of individuals and the grieving families.Practicing dentists are expected to maintain accurate dental records and extend their co-operation to the investigating agencies whenever required.The dental expert is expected to render his knowledge and skills with truth, impartiality,thoroughness and accuracy.

\section{REFERENCES:}

[1] Sylvie Louise Avon, DMD, MSc Forensic Odontology:The Roles and Responsibilities of the Dentist J Can Dent Assoc 2004; 70(7):453-8

[2] R.David .Senn, G.Paul .Stimson,Forensic Dentistry,Second Edition, $5^{\text {th }}$ chapter,D.Kimberley Molina forensic medicine and human identification p-61-63.

[3] A. Pretty, and D. Sweet, A look at forensic dentistry - Part 1: The role of teeth in the determination of human identity British Dental Journal, volume 190, NO. 7, APRIL 142001 359-366

[4] Pisha Pittayapat, Reinhilde Jacobs, Eddy De Valck, Dirk Vandermeulen, Guy Willems forensic odontology in the disaster victim identification process JFOS. July 2012, Vol.30, No.1 Pag 1-12

[5] 5.J. Augustine, S.R. Barpande, J.V. Tupkari, cheiloscopy as an adjunct to forensic identification: a study of 600 individuals J Forensic Odontostomatol 2008;27:2:44-52)

[6] A.Corte-Real, M.J.Anjos, D.N. Vieira, J.J. Gamero, The Tooth for Molecular Analysis and Identification :a Forensic Approach JFOS. July 2012, Vol.30, No.1 p 22-28

[7] BR Rothwell, Bite marks in forensic dentistry: a review of legal, scientific issues JADA 1995;126;223-232

[8] S.A.Shinde, M.K, Malve, Chauhan Prabha, and M.V, Garad, Nanotechnology and Forensic Science Nanotechnology and Nanoscience, Vol. 1, Issue 1, 2010 PP-19-21

[9] 9.A.B,Fan Yang A. Reinhilde Jacobs Guy Willems Dental age estimation through volume matching of teeth imaged by conebeam CT, Forensic Science International 159S (2006) S78-S83

[10] 10.Guy Williams A review of the most commonly used dental age estimation techniques,J Forensic Odontostomatol 2001,19,9-17

[11] 11.P E M Dias,a* T L Beaini,a R F H Melani Age estimation from dental cementum incremental lines and periodontal disease J Forensic Odontostomatol 2010;28:1:13-21

[12] 12.G.G. Stott, R.F. Sis and B.M. Levy Cemental Annulation as an Age Criterion in Forensic Dentistry J DENT RES 1982 61 :814817

[13] 13.L. Lima,a Y. da Costa,b R. Tinoco,a P. Rabello,b E. Daruge Junior.a Stature estimation by carrea's index and its reliability in different types of dental alignment J Forensic Odontostomatol 2011;29:1:7-13 